\title{
Mögliche Ansätze für die Primärprävention von Adipositas
}

D. Fäh

Die Zahl übergewichtiger Menschen nimmt weltweit zu. Besonders ausgeprägt ist diese Zunahme bei Kindern und Jugendlichen. Dieser Trend macht auch vor der Schweiz nicht halt, wo laut letzten Untersuchungen bereits jedes 5 . Kind zu dick ist. Der Preis, welchen künftige Generationen dafür werden zahlen müssen, lässt sich kaum abschätzen. Um die Folgen einzudämmen, sind Massnahmen gefragt. Diese müssten früh und tief greifen und möglichst fest und breit im gesellschaftlichen Leben verankert sein. Denkbare Ansatzpunkte fänden sich in den Bereichen Erziehung und Ausbildung. Auch staatliche Eingriffe in Werbung und Stadtplanung sowie Besteuerung und Nährwertdeklaration von Lebensmitteln stünden zur Diskussion.

\section{Übergewicht macht vor der Schweiz nicht halt}

Die XXL-Gesellschaft stellt ein zunehmendes Gesundheitsproblem dar. In den Vereinigten Staaten leiden bereits zwei Drittel der Bevölkerung an Übergewicht und Adipositas [1]. Im Jahr 2000 kostete Fettsucht in den USA 400000 Menschen das Leben; das entspricht 17 Prozent aller Sterbefälle. Nur noch knapp hinter den Tabaktodesopfern, ist die Krankheit auf bestem Wege, zur Nummer eins unter den vermeidbaren Todesursachen $\mathrm{zu}$ avancieren [2]. Epidemien scheren sich bekanntlich nicht um Landesgrenzen, weshalb längst auch Europa davon betroffen ist. Länder wie Italien und Deutschland sind nicht mehr weit von amerikanischen Verhältnissen entfernt, und auch hierzulande zeigt der Trend klar in die gleiche Richtung. In der Schweiz sind rund zwei Millionen Menschen zu dick, was 37\% der Gesamtbevölkerung entspricht [3]. Trotz dieser alarmierenden Zahlen hielten es Politiker bisher nicht für nötig, konkrete Präventionsvorschläge und -massnahmen in der breiten Öffentlichkeit zu diskutieren.

\section{Immer mehr Kinder betroffen}

Korrespondenz:

Dr. med. David Fäh

Institut universitaire de médecine

sociale et préventive (IUMSP)

Rue du Bugnon 17

CH-1005 Lausanne
Ignorieren oder Unterschätzen dieser globalen Epidemie würde jedoch fatale Folgen nach sich ziehen. Insbesondere deshalb, weil zunehmend junge Menschen davon betroffen sind. Die stei- gende Zahl übergewichtiger Kinder lässt vermuten, dass das Auftreten heutiger Zivilisationskrankheiten lediglich die Spitze des Eisbergs darstellt. Jüngste Untersuchungen müssten alarmieren: Eines von fünf Kindern in der Schweiz leidet an Übergewicht oder Fettleibigkeit (Adipositas). Dieser Anteil hat sich in den letzten 20 Jahren verdreifacht [4]. Es wächst also eine Generation heran, die ein beträchtliches Risiko trägt, in ihrem Leben an einem vermeidbaren Herzinfarkt, Hirnschlag oder Krebs zu erkranken oder daran zu sterben.

\section{Suche nach dem Sündenbock}

Wer mehr isst, als er verbraucht, nimmt zu. So einfach und plausibel diese Feststellung klingen mag, so schwer tun sich Experten bei der Entscheidung darüber, was nun wirklich dick macht. Viele von ihnen unterliegen dem menschlichen Bedürfnis, einfache Erklärungen für schwierige Probleme zu suchen. Während Wissenschaftler in den 90er Jahren vor allem Fett an den Pranger stellten, scheinen sie sich heute auf Kohlenhydrate [5] eingeschossen zu haben. Weit entscheidender für das Körpergewicht eines Kindes ist jedoch die Summe der Faktoren, welche seine Energiebilanz aus dem Gleichgewicht bringen können: Auf der Einnahmeseite spielen sicherlich billige, überall und allzeit verfügbare energiereiche Nahrungsmittel eine (ge-)wichtige Rolle, wobei hier zunehmend gezuckerte Süssgetränke [6] ins Schussfeld der wissenschaftlichen Kritik geraten. Auf der Ausgabeseite fällt die steigende Bewegungsarmut der jüngsten Generation ins Auge: weniger Sport in Schule und Freizeit, eingeschränkte Bewegungsmöglichkeiten in Stadt und Agglomeration. Hingegen ziehen Fernseher und Computer Kinder und Jugendliche immermehr und länger in ihren Bann. In mehreren Studien war die vor dem Fernseher verbrachte Zeit derjenige Faktor, welcher am stärksten mit dem Risiko für das Auftreten von Übergewicht verbunden war [7]. Das verwundert nicht weiter, schliesslich ist 
mit dem TV-Konsum neben Bewegungsarmut auch Knabbern und Berieselung mit Nahrungsmittelwerbung verbunden.

\section{Gesellschaftlicher Wandel mitverant- wortlich?}

Hohe Scheidungsraten und steigende Anforderungen in der Berufswelt sorgen auch in der Schweiz dafür, dass traditionelle Familienstrukturen seltener werden. Während Eltern ihrem Nachwuchs früher mit der gemeinsamen Zubereitung und dem Genuss eines Mahls Esskultur vermittelten, bleibt heute dieser Luxus vielen Kindern verwehrt. Da hierzulande Tagesschulen und Mittagstische dünn gesät sind, ersetzen häufig Fast food und energiereiche Snacks und Getränke eine regelmässige und vollwertige Mahlzeit. Dass die Vorbildfunktion der Eltern tatsächlich wesentlich darüber entscheidet, ob ihr Kind im späteren Leben mit Gewichtsproblemen zu kämpfen hat, scheint unbestritten [8]. Der wissenschaftliche Beweis, dass Kinder aus Patchworkfamilien eher zu Übergewicht neigen, steht jedoch noch aus.

\section{Übergewichtige Kinder tragen doppelt schwer}

Als Kind dick zu sein gehört wohl zu den härtesten Lebensproben überhaupt. Von Klassenkameraden gehänselt, leidet das Selbstbewusstsein. Häufig können oder wollen übergewichtiger Kinder nicht am Turnunterricht teilnehmen. Die ausserschulischen Aktivitäten beschränken sich oft auf Fernsehkonsum und Videospielen. Als vermeintlicher Ausweg bleibt oft nur die Flucht in die soziale Isolation, von wo aus sie Trost im Essen suchen. Aus diesem Kompensationsmechanismus entwickeln sich nicht selten hartnäckige Essstörungen, die mit dem Übergewicht ins Erwachsenenalter mitgenommen werden. Der Weg aus dem Teufelskreis ist steinig und übersät mit Rückschlägen. Und mit jedem Kilo zuviel sinkt die Chance auf ein gesundes Körpergewicht. Wer im Bereich Adipositastherapie Erfahrungen sammeln durfte, weiss, dass die Aussichten auf Erfolg bescheiden sind, insbesondere bei lange bestehendem und ausgeprägtem Übergewicht. Leider gilt dies oft auch für stationäre Therapie. Wieder in das ursprüngliche soziale Gefüge, in die alten Ess- und Bewegungsgewohnheiten zurückgekehrt, stellt sich nicht selten auch das ursprüngliche Körpergewicht wieder ein.

\section{Status quo im Kampf gegen Übergewicht}

Im Bemühen um die Adipositastherapie und -prävention sind Ansätze erkennbar. So wurde beispielsweise 2002 «Suisse Balance» [9], ein Gemeinschaftsprojekt des Bundes und der Gesundheitsförderung Schweiz [10] (Kantone), ins Leben gerufen. Im gleichen Jahr wurde der schweizerische Fachverein «Adipositas im Kindes- und Jugendalter (AKJ)» [11] gegründet, und auch der Bund ist mit «Bildung und Gesundheit» [12] aktiv geworden. Im Bereich Primärprävention existieren Projekte wie «Nutrikid» [13], «5 am Tag» [14] oder die Netzwerke «hepa» [15] und «Nutrinet» [16]. Doch so erfreulich die letzten Entwicklungen auch sind, sie reichen bei weitem nicht! Zudem scheitern viele Bemühungen und sinnvolle Projekte an den äusserst knappen Mitteln. Das Bundesamt für Gesundheit (BAG) stellt 800000 Franken jährlich für Gesundheitsförderung und Prävention im Ernährungsbereich zur Verfügung ein Tropfen auf den heissen Stein. Zum Vergleich: Für die Aidsprävention stehen jährlich 9 Mio., für die Alkoholprävention (über den Alkoholzehntel finanziert) 24 Mio. Franken bereit. Ein BAG-Fonds zur Bekämpfung des Rauchens stellt pro Jahr rund 20 Mio. Franken zur Verfügung.

\section{Primärprävention als Schlüssel zum Erfolg?}

Um den Trend zu immer mehr dicken Kindern $\mathrm{zu}$ brechen und umzukehren, bedarf es tiefgreifender, struktureller Massnahmen, wobei insbesondere der Gesetzgeber gefragt ist. Die Massnahmen müssten so früh wie möglich, das bedeutet bereits vor der Entstehung von Übergewicht, greifen und alle ansprechen, schliesslich ist niemand davor gefeit. Darin sind sich die meisten Experten einig: Besonders in der Adipositasvorbeugung ist die Primärprävention am sinnvollsten und kosteneffizientesten [17, 18]. Sie muss Anreize und ein Umfeld schaffen, um die Energiebilanz zu verbessern. Im Falle von jungen Menschen hiesse dies, Rahmen zu schaffen für eine ausgewogene Ernährung und ausreichende körperliche Aktivität sowie die Förderung einer gesunden Ess- und Bewegungskultur, oder anders ausgedrückt: die Entgiftung der Umwelt [19]. Nicht zu vergessen eine psychologische Unterstützung in einer immer hektischeren und stärker fordernden Schulwelt. Absolut entscheidend ist der Miteinbezug des gesamten sozialen Umfeldes, das heisst von Eltern, 
Lehrern und sonstigen Bezugspersonen. Im Detail stünden folgende Massnahmen zur Diskussion:

\section{Ausbildung und Erziehung}

- Sensibilisierung, Aus- und Weiterbildung von Lehrern und Ärzten, Psychologen/-innen, Ernährungsberater/innen und Köchen/ -innen sowie von Tagesmüttern und Sozialarbeitern/-innen;

- Ausweitung des Schullernstoffs in den Bereichen Ernährungslehre, Lebensstil und Gewichtskontrolle; Einführung eines Schulfachs «Gesünder leben»;

- Intensivierung des Sportunterrichts und Vermittlung eines positiven Körpergefühls; eventuell gesonderter Unterricht für übergewichtige und gefährdete Kinder;

- Gesundheitsförderung über TV, Radio, Printmedien und Internet, in Einkaufsläden, Kantinen und während der Schulpausen.

\section{Gesetzliche Regelungen}

- zusätzliche Besteuerung von Elementen, die körperliche Aktivität vermindern, wie motorisierte Fahrzeuge, Fernseher und Videospiele; Einführung einer Maut für den Innenstadtverkehr («Roadpricing», wie es derzeit für die Londoner Innenstadt existiert);

- staatliche Förderung des öffentlichen Verkehrs, von Fahrrädern und Fitnessgeräten;

- Regelung von Werbung während Kindersendungen, begleitet von Massnahmen, die den TV-Konsum von Kindern reduzieren sollen;

- Steuererleichterungen für Unternehmen, die körperliche Aktivität und eine gesunde Ernährung ihrer Mitarbeiter fördern; staatliche Förderung für Sportvereine, Schwimmbäder, Fitnesscenter und Parks;

- Auflagen für Städteplanung bezüglich Gehund Radwegen, Grünflächen, Spiel- und Sportplätzen; Schaffung sicherer und kinderfreundlicher Schulwege;

- Einschränkung von Nahrungsmittel- und Getränkeautomaten in Schulen; Subventionierung des Früchte- und Gemüseangebots in Schulen sowie einer gesunden, warmen Mahlzeit;

- Steuern auf Esswaren und Getränken mit hohem Zucker- und/oder Fettgehalt; Besteuerung des Angebots von Fast-food-Kon- zernen, ergänzt durch gesetzliche Auflagen bezüglich Vermarktung und Portionsgrösse; finanzielle Anreize für gesundes Nahrungsmittelangebot in Restaurants;

- Vorschriften für Verpackungsdesign und Einheitsgrössen für Esswaren sowie Angaben über Kalorien, Fett- und Zuckergehalt (oder in Form eines vereinfachten Punktesystems) in Einkaufsläden und Restaurants; Erarbeiten und Verteilen von Gütesiegeln für kalorienarme und nährstoffreiche Produkte und Menüs (siehe «Fourchette verte» [20]).

\section{Adipositasprävention als Bestandteil einer schweizerischen Ernährungspolicy}

- Ähnlich wie bei der Alkohol und Tabakprävention müssen Präventionskonzepte erarbeitet werden mit klaren Strategien und definierten Meilensteinen;

- feste Zuordnung der Mittel aus den Lenkungsabgaben für Bildung und Forschung und Erarbeitung von Konzepten; Bestimmung der Forschungsschwerpunkte;

- Erlassen eines nationalen Präventionsgesetzes; Entwicklung einer nationalen Adipositaspräventionskampagne.

Mögen viele der oben erwähnten Vorschläge fraglich erscheinen, so ist deren Erwägung und Prüfung dringlicher denn je. Nur die Schaffung eines entsprechenden Umfeldes kann der wachsenden Epidemie «Übergewicht» wirksam Einhalt gebieten.

\section{Literatur}

1 Flegal KM, Carroll MD, Ogden CL, Johnson CL. Prevalence and trends in obesity among US adults, 1999-2000. JAMA 2002;288(14):1723-7.

2 Mokdad AH, Marks JS, Stroup DF, Gerberding JL. Actual causes of death in the United States, 2000. JAMA 2004;291(10):1238-45.

3 Schweizerische Gesundheitsbefragung 2002. Neuchâtel; 2003.

4 Zimmermann MB, Gubeli C, Puntener C, Molinari L. Detection of overweight and obesity in a national sample of 6-12-y-old Swiss children. Am J Clin Nutr 2004;79(5):838-43.

5 Jenkins DJ, Kendall CW, Marchie A, Augustin LS. Too much sugar, too much carbohydrate, or just too much? Am J Clin Nutr 2004;79(5):711-2.

6 Bray GA, Nielsen SJ, Popkin BM. Consumption of high-fructose corn syrup in beverages may play a role in the epidemic of obesity. Am J Clin Nutr, 2004;79(4):537-43. 
7 Crespo CJ, Smit E, Troiano RP, Bartlett SJ, Macera CA, Andersen RE. Television watching, energy intake, and obesity in US children: results from the third National Health and Nutrition Examination Survey, 1988-1994. Arch Pediatr Adolesc Med 2001;155(3):360-5.

8 Hood MY, Moore LL, Sundarajan-Ramamurti A, Singer M, Cupples LA, Ellison RC. Parental eating attitudes and the development of obesity in children. The Framingham Children's Study. Int J Obes Relat Metab Disord, 2000;24(10): $1319-25$

9 www.suissebalance.ch [accessed 10 Sept. 2004].

10 www.promotionsante.ch [accessed 10 Sept. 2004]

11 www.minuweb.ch [accessed 10 Sept. 2004].

12 www.bildungundgesundheit.ch [accessed 10 Sept. 2004].
13 www.nutrikid.ch [accessed 10 Sept. 2004].

14 www.5amtag.ch [accessed 10 Sept. 2004].

15 www.hepa.ch [accessed 10 Sept. 2004].

16 www.nutrinet.ch [accessed 10 Sept. 2004].

17 Nestle M, Jacobson MF. Halting the obesity epidemic: a public health policy approach. Public Health Rep 2000;115(1):12-24.

18 Obesity, prevention and managing the global epidemic. Report of a WHO consultation on obesity, 3-5 June 1997. World Health Organization; ed. 1998.

19 Ebbeling CB, Pawlak DB, Ludwig DS. Childhood obesity: public-health crisis, common sense cure. Lancet 2002;360(9331):473-82

20 www.fourchetteverte.ch [accessed 10 Sept. 2004]. 